\title{
Wet-electrospun CuNP/carbon nanofibril composites: potential application for micro surface-mounted components
}

\author{
Ashraf A. Ali • Awad Kh. Al-Asmari
}

Received: 7 February 2011/ Accepted: 12 October 2011/Published online: 1 November 2011

(c) The Author(s) 2011. This article is published with open access at Springerlink.com

\begin{abstract}
Wet electrospinning of polyacrylonitrile (PAN) and dimethylformamide (DMF) with copper nanoparticles (CuNP) at different concentrations from 0.2 to $1 \mathrm{wt} \%$ have been studied under certain spinning conditions. A specific coagulating water bath has been used to collect different fibroses and fibril diameters, the effect of spinning height on the produced nanofiber and CuNP/PAN nanofibril composites have been studied from 1 to $7 \mathrm{~cm}$ heights. A minimum average diameter of $64 \mathrm{~nm}$ has been reported at 7 -cm spinning height. Two heat treatment steps have been used to enhance the electrical properties of CuNP/PAN nanofibril composites. SEM has been used to study the morphological characteristics of the electrospun nanofibroses membranes. Preliminary electrical measurements using 4-point probing system showed a noticeable improvement in the electrical conductivity of the produced nanofibril composite membranes. Also, electrical property of a single CuNP/carbon nanofibril composite has been theoretically calculated based on Lichtenecker formula. The produced membranes have been used to build a micro surface-mounted components (MSMC) such as Micro Field Effect Transistor (MFET). A high transconductance has been reported for such a device which will open the door for many promising applications especially in Electronics and Biomedicine.
\end{abstract}

A. A. Ali $(\bowtie)$

Mechanical Engineering Department, Faculty of Engineering,

Salman Bin Abdul-Aziz University, P.O. Box 655,

AlKharj 11942, Saudi Arabia

e-mail: afattah@ksu.edu.sa

A. Kh. Al-Asmari

Electrical Engineering Department, Faculty of Engineering,

King Saud University, Riyadh, Saudi Arabia
Keywords Wet Electrospinning · CuNP · Carbon · Nanofibril composites · Electrical Properties

\section{Introduction}

Electrospinning processes has been used to produce fibers from nanometer range to conventional fiber sizes. Reneker and Chun (1996) started to use the electrospinning as a technique to produce small diameter fibers. In this paper they used poly (p-phenylene terephthalamide) to produce Kevlar fibers with superior physical properties by dry-jet-wet spinning technique assisted by electrostatic charges. Monofilament Kevlar fibers ranged from 13 to $18 \mu \mathrm{m}$ were produced under an applied voltage of $12-18 \mathrm{kV}$ and distance of $3 \mathrm{~cm}$ in a vertical position. Doshi and Reneker (1995) described the electrospinning process, the processing conditions, fiber morphology, and some possible uses of electrospun fibers from polyethylene oxide (PEO) of molecular weight $(\mathrm{Mw})=145,000 \mathrm{~g} / \mathrm{mol}$, surface tension 61 dynes $/$ $\mathrm{cm}$, conductivity $=400 \mu \mathrm{s} / \mathrm{cm}$ and solution viscosity $=$ $800-4,000 \mathrm{cP}$. They found that; for viscosity below $800 \mathrm{cP}$, polymer solution was too dilute to form a stable jet and the jet broke. At a viscosity above $4,000 \mathrm{cP}$ it was difficult to form fibers due to drying of the solution at the tip. Fang and Reneker (1996) studied a concentrations effect on the produced nanofiber. Concentrations from 1 to $7 \%$ by weight were used. They concluded that as the concentration increases the required voltage to start spinning is increased; for example, for concentration of 2.5 and $5 \%$ by weight the required voltages to start were 9 and $12 \mathrm{kV}$ respectively. This paper also showed that the diameter of the jet increased as the distance between the spinneret and the target decreased. Ali et al. (2002) optimized the electrospinning process with PAN polymer solution system to produce 
$100 \mathrm{~nm}$ fiber diameters. They studied the effect of the heat treatment on the morphology of the electrospun PAN fibers. Also, a demonstration using the electrospinning process to produce continuous yarn from polyacrylonitrile polymer solutions has been presented, which will open the door for linear, planar and 3D fiber assemblies for nanocomposites specially for carbon-carbon composites. Electrospun nanofibers are characterized by its high surface area; this characterization showed a different behavior for the nanosized fibers such as super absorbent characteristics for electrospun polyacrylamide (PAM) nanofibers (Ali 2008) and nanoreinforcement size effect with multiwall carbon nanotubes (MWCNT) (Ko et al. 2003) and graphite nanoplatelet (Mack et al. 2005) which has been correlated to the unusual behavior of molecular chains on the surface of nanoscale fibers. It is well known that, atoms at a free surface experience a different local environment than do atoms in the bulk of a material; as a result, the energy associated with these atoms will, in general, be different from that of the atoms in the bulk more over the heat dissipation of these higher surface area of such fibroses materials will be much more than for even thin bulk materials. Also, the expected properties of a combination of two nanosized structures will result in superior properties. Bal (2010) showed an addition of $1 \mathrm{wt} \%$ of carbon nanofibers brought improvement in electrical properties of epoxy composite. Spitalsky et al. (2010) studied nanotube/ polymer composites in which the presence of interconnected nanotube network results to a dramatic increase of their electrical conductivity. Also, nanocomposites based on Poly methyl methacrylate (PMMA) and MWCNT as a filler show a significant enhancement in the electrical conductivity. Kim et al. (2004) reported a value of 3,000 $\mathrm{S} / \mathrm{m}$ at $0.4 \mathrm{wt} \%$ for extremely low percolation threshold (0.003 wt $\%)$ prepared by the solution mixing method. Furthermore, the addition of MWCNT to the polyester matrix PC by the melt mixing technique induces a dramatic increase of about 16 orders of magnitude in the maximum conductivity; reaching the value of $1,000 \mathrm{~S} / \mathrm{m}$ at $15 \mathrm{wt} \%$ (Pötschke et al. 2004). According to Koerner et al. (2005) a maximum electrical conductivity of $2,000 \mathrm{~S} / \mathrm{m}$ is observed for Polyurethane (PU) filled with $27 \mathrm{wt} \%$ MWCNT using the solution casting technique. The same conductivity value has been achieved for single wall carbon nanotube (SWCNT)/PAN composite prepared by solution mixing at $15 \mathrm{wt} \%$ loading. Composite fibers SWCNT/polyethyleneimine (PEI) prepared by means of the coagulation fiber spinning process exhibit a considerably high maximum electrical conductivity of $2 \times 10^{4} \mathrm{~S} / \mathrm{m}$. An enhancement of maximum electrical conductivity compared to the neat polymer, is observed in nanocomposites based on conducting polymers such as polypyrrole (PPY). For example a fourfold increase of electrical conductivity at $50 \mathrm{wt} \%$ loading is obtained for composites prepared by an in situ polymerization process (Fan et al. 1999). Recently, polyphenylene sulfide plastic (PPS)-based composites containing $5.4 \mathrm{wt} \%$ MWCNT have been reported to exhibit a maximum electrical conductivity of about $1,000 \mathrm{~S} / \mathrm{m}$ at relatively low percolation thresholds $(0.396 \mathrm{wt} \%)$. As a general statement, the excellent electrical properties of composites containing MWCNT are promising for the design of low cost polymer composites for numerous future applications (Spitalsky et al. 2010). Also, nanodiamond nucleation has been reported for carbon nanomaterials below the required pressure and temperature for similar bulk materials (Sun et al. 2005; Guillou et al. 2007; Ali and Rutledge 2009). Ali and Al-Asmari (2010) reported a nanodiamond nucleation due to the interaction between MWCNT and electrospun PAN nanofiber during carbonization process that interaction results in a formation of $3 \mathrm{D}$ structure as well as an enhancement in the electrical properties of the produced fabrics. The hypothesis behind this research work is an attempt to continue a work that has been started by Ali and Rutledge (2009) by utilizing the high surface energy associated with the electrospun PAN nanofibers along with different nanomaterials, such as carbon nanotube (Ali and Al-Asmari 2010) and copper nanoparticles (present study), as a cohesive bond to build a firm and strong precursor superior nanofibril hybrid fabrics. Also, to get the use of the high degree of entanglement inside electrospun nanofibrous structure to gain a higher degree of flexibility in the overall produced fabrics. Also, the present study is an attempt to enhance the electrical properties of electrospun PAN nanofibers through two steps; the first step is forming graphitic structure during the heat treatment and the second step is adding nanocopper particles to the polymer solution during electrospinning and then heat treat the whole nanofibril composite aiming to form 3D ordered structure as a result of the interaction between the both carbon nanofibers and copper nanoparticles. These new conductive nanofibril composite flexible and strong fabrics are characterized by its higher dissipation of heat more than traditional materials as a result of high surface area as well as the possibility to control its conductivity values so that it can be used from nonconductive as a lightning strike shield to semiconductive as surfacemounted components. Finally, to highlight on the difference in electrical properties between the fabric and its constituents by studying the effect of copper nanoparticles weight concentration on morphological and electrical characteristics of a single nanofibril composites versus their in-plan randomly oriented nanofibroses composite fabrics. 


\section{Experimental work}

\section{Materials}

Polyacrylonitrile (PAN) of $150,000 \mathrm{~g} / \mathrm{mol}$ molecular weight from Aldrich was used with $10 \%$ weight concentration in dimethylformamide (DMF) to form a polymer solution after hot stirring for $3 \mathrm{~h}$ at $60^{\circ} \mathrm{C}$ to ensure a complete solubility.

Copper nanopowder $<50 \mathrm{~nm}$ (TEM), 99.5\% from Sigma-Aldrich catalog number CAS 7440-50-8 has been well dispersed (after 24 sonication hours) inside the PAN/ DMF polymer solution with five different concentrations $0.2,0.4,0.6,0.8$ and 1 by weight $\%$.

\section{Electrospinning}

The PAN/DMF-CuNP polymer solution was poured to fill a clean glass pipette of $2 \mathrm{ml}$ volume and $1 \mathrm{~mm}$ orifice diameter, and the pipette was placed vertically in drilled PMMA stand, PS/FC60R02.0-22 Model GLASSMAN High Voltage of $60 \mathrm{kV}$ maximum positive voltage and three digits milliamps current accuracy has been applied to the polymer solution inside the pipette. A coagulating PMMA bath of 30 (length) $\times 20$ (width) $\times 20$ (depth) in $\mathrm{cm}$ has been filled with distilled water to the depth of $15 \mathrm{~cm}$. A grounded wire has been merged inside the coagulating bath. Four different spinning heights $(\mathrm{H}) 1,3$, $5,7 \mathrm{~cm}$ have been selected to collect CuNP/PAN fibroses structures. These heights were measured precisely from the orifice of the pipette to the surface of water. The electrospun fibroses membranes were collected using a manual fishing assisted by aluminum foils of $15 \times$ $15 \mathrm{~cm}$.

\section{Heat treatments}

The collected fibroses membrane was placed in a ceramic boats size 5 then placed in A CARBOLITE tube furnace of $75 \mathrm{~mm}$ diameter $600 \mathrm{~mm}$ effective length and $1,100^{\circ} \mathrm{C}$ maximum temperature.

\section{Stabilization}

The tube furnace is set to reach $220^{\circ} \mathrm{C}$ in open environment and the samples inside the ceramic boats were placed inside the tube furnace for $20 \mathrm{~min}$. Then, samples have been hot pressed under 5 metric tons to activate the surface energy associated with the nanofibers to produce flexible and strong $\mathrm{CuNP} /$ carbon nanofibril composite fabrics (Ali and Rutledge 2009).

\section{Carbonization}

The tube furnace is set to reach $750^{\circ} \mathrm{C}$ with nitrogen dynamic flow of $5 \mathrm{ml} / \mathrm{min}$ and the samples were placed inside the tube furnace for $1 \mathrm{~h}$.

\section{Electrical conductivity measurements}

A four point probing system has been used to measure the conductivity of the carbonized membranes. Rule of mixture along with Lichtenecker formula (Zakri et al. 1998) and Uvarov (2000) approach have been used to estimate the electrical conductivity values for both $\mathrm{CuNP} /$ carbon single nanofibril composites and nonwoven fibroses fabrics.

\section{Results and discussion}

Effect of spinning height on wet-electrospun fiber diameter

The morphological behavior of the nanofibers before and after adding CuNP has been investigated using SEM. As shown in Figs. 1, 2 the effect of spinning heights on the collected wet-electrospun fiber diameters has been studied and summarized. Each SEM has been divided into four equal quarters and same reading numbers from each quarter have been measured using a simple computer software, then the average of all readings has been calculated as well as standard deviation. Table 1; Fig. 2 summarize the results. It is obvious from Fig. 2 that as spinning height increases the average diameter of the collected PAN fibers decreases as well as its standard deviation which is consistent with other publications (Reneker and Chun 1996; Ali 2008; Ko et al. 2003) and it has been explained due to the increase of the traveling path for the whipping movement of the unstable zone which permits both more evaporation of the solvent and more reduction in the diameter of the terminated jet.

\section{Effect of CuNP wt $\%$ on wet-electrospun fiber diameter}

The effect of CuNP wt $\%$ on the produced nanofibril composites has been studied as well. Figure 3 shows the SEM images of various CuNP wt\% collected samples at 7-cm spinning height.

Each SEM has been divided into four equal quarters and same reading numbers from each quarter have been measured using a simple computer software then the average of all readings has been calculated as well as standard deviation. Table 2; Fig. 4 summarize the results.

As shown in Fig. 4 for both heights as CuNP wt $\%$ increases the nanofibril composite diameter increases as 


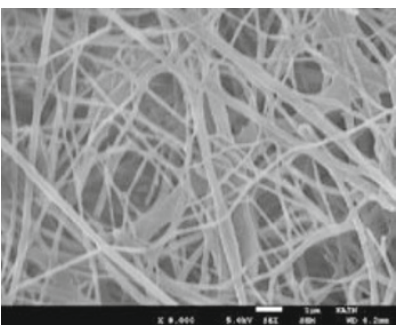

$\mathrm{H}=\mathbf{1} \mathrm{cm}$

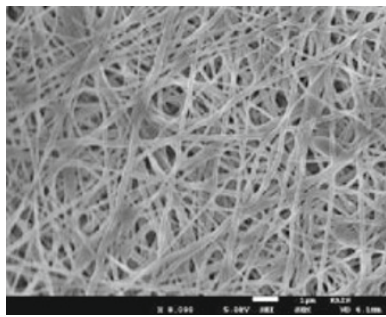

$\mathrm{H}=\mathbf{5} \mathrm{cm}$

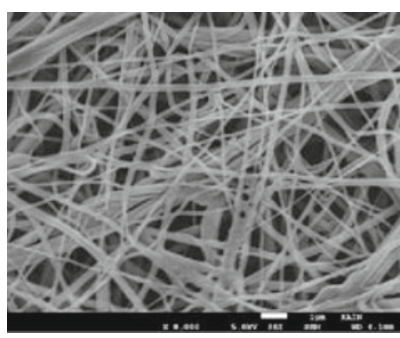

$\mathbf{H}=\mathbf{3} \mathbf{c m}$

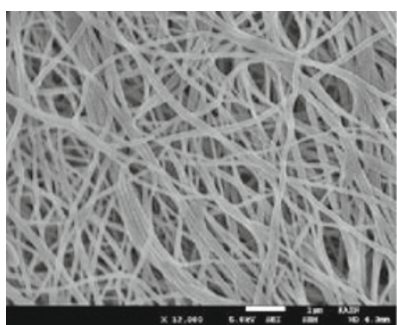

$\mathrm{H}=7 \mathrm{~cm}$
Fig. 1 SEM Images of as wet-electrospun PAN fibroses membranes

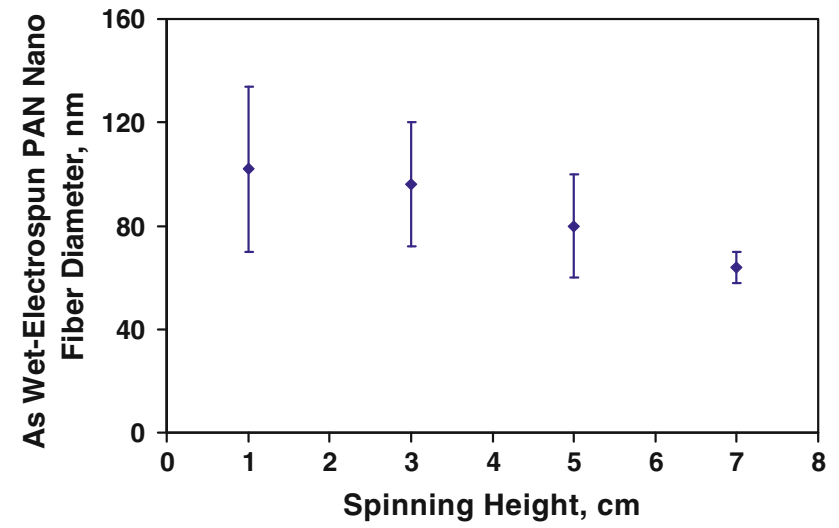

Fig. 2 Effect of spinning height on PAN nanofiber diameter

Table 1 Effect of spinning height on fiber diameter

\begin{tabular}{lll}
\hline Spinning height & Fiber diameter & $\begin{array}{l}\text { Standard deviation } \\
H(\mathrm{~cm})\end{array}$ \\
\hline 1 & $d(\mathrm{~nm})$ & STDV $(\mathrm{nm})$ \\
3 & 102 & 32 \\
5 & 96 & 24 \\
7 & 80 & 20 \\
\hline
\end{tabular}

well as the fibril distribution represented in standard deviation values. It is also noticed that as spinning height decreases the increase in diameter was maintained. It is worthy to report here that based on experimental observations it has been noticed that the values of voltage and current could not be fixed for all spinning heights and

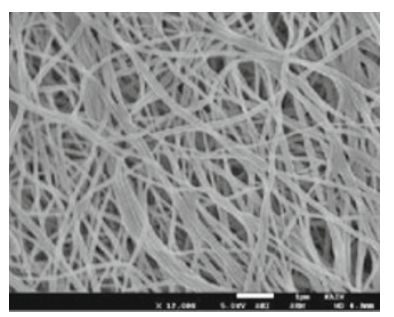

0 wt \% CuNP - H = $7 \mathrm{~cm}$

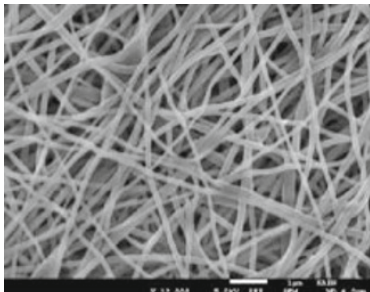

$0.4 \mathrm{wt} \% \mathrm{CuNP}-\mathrm{H}=7 \mathrm{~cm}$
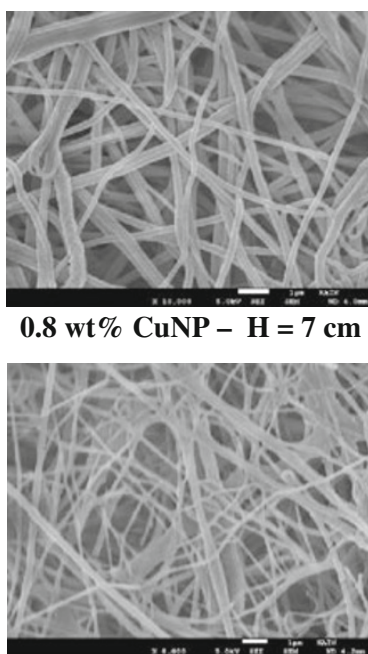

$0 \mathrm{wt} \% \mathrm{CuNP}-\mathrm{H}=1 \mathrm{~cm}$
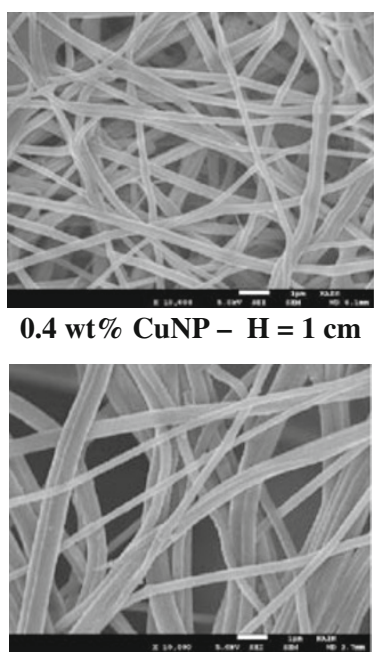

$0.8 \mathrm{wt} \% \mathrm{CuNP}-\mathrm{H}=1 \mathrm{~cm}$

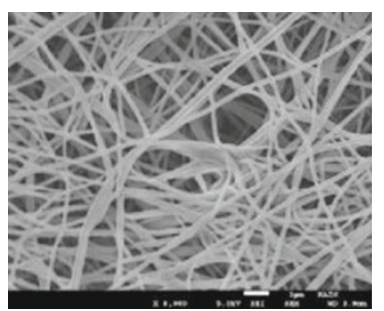

$0.2 \mathrm{wt} \% \mathrm{CuNP}-\mathrm{H}=7 \mathrm{~cm}$
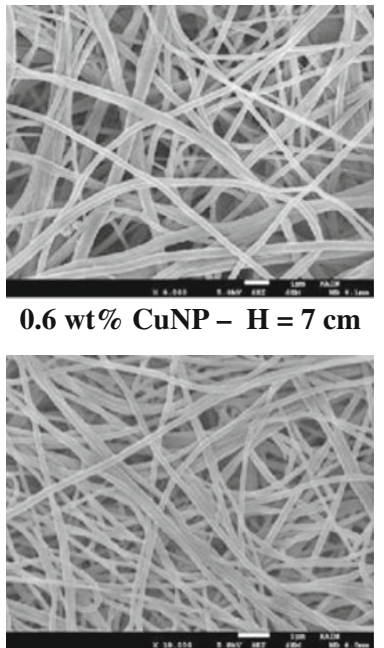

$1 \mathrm{wt} \% \mathrm{CuNP}-\mathrm{H}=7 \mathrm{~cm}$

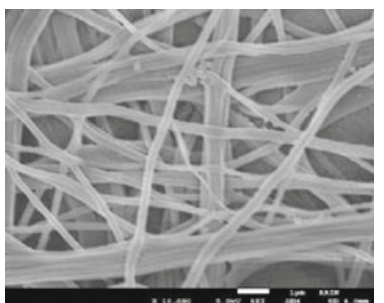

$0.2 \mathrm{wt} \% \mathrm{CuNP}-\mathrm{H}=1 \mathrm{~cm}$
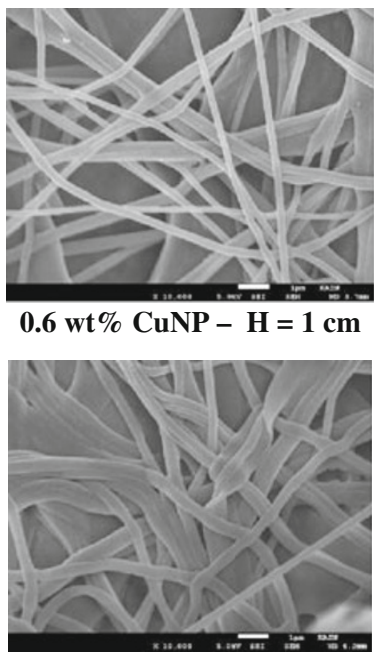

$1 \mathrm{wt} \% \mathrm{CuNP}-\mathrm{H}=1 \mathrm{~cm}$

Fig. 3 SEM Images of as wet-electrospun CuNP/PAN fibroses membranes 
Table 2 Effect of spinning height on fibril diameter

\begin{tabular}{|c|c|c|c|c|}
\hline \multirow[t]{2}{*}{ CuNP wt $\%$} & \multicolumn{2}{|c|}{$H=1 \mathrm{~cm}$} & \multicolumn{2}{|c|}{$H=7 \mathrm{~cm}$} \\
\hline & $d(\mathrm{~mm})$ & STDV (mm) & $d(\mathrm{~mm})$ & STDV $(\mathrm{mm})$ \\
\hline 0 & 102 & 32 & 64 & 6 \\
\hline 0.2 & 207 & 37 & 136 & 11 \\
\hline 0.4 & 207 & 36 & 157 & 14 \\
\hline 0.6 & 206 & 43 & 200 & 24 \\
\hline 0.8 & 227 & 36 & 202 & 22 \\
\hline 1.0 & 293 & 45 & 202 & 25 \\
\hline
\end{tabular}

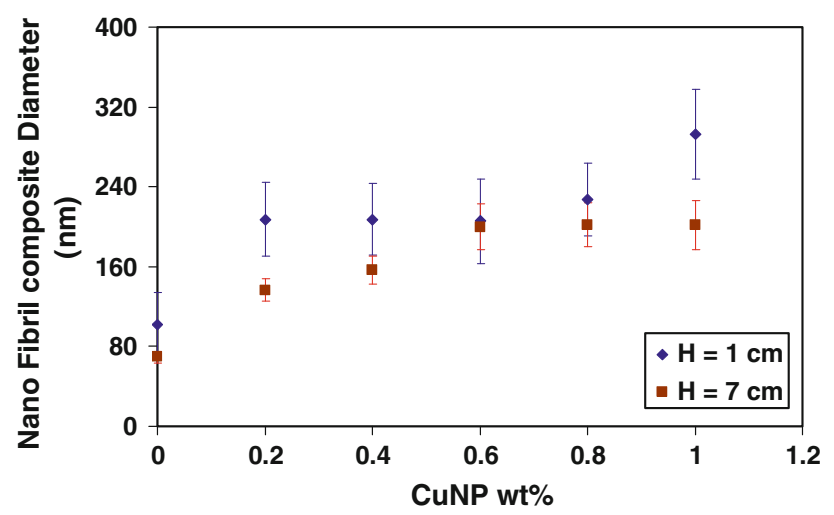

Fig. 4 Effect of CuNP Wt\% on wet-electrospun CuNP/PAN nanofibril composite diameter

CuNP wt \%, and it was recorded for each case and the voltage range was between 16 and $20 \mathrm{kV}$ and for current it was from 0.001 to $0.004 \mathrm{~mA}$. It is obvious that the effect of both spinning height and CuNP wt\% on the applied volt and current requires more investigation especially below 1-cm spinning height which has been found as the key parameters of the formation of coil- like structures that will be discussed separately in another research publication.

Heat treatment

\section{Stabilization and carbonization}

A formation of $\mathrm{CuNP} /$ carbon nanofibril composites of different CuNP wt\% concentration aim to enhance the electrical conductivity of the whole fabrics. A high resolution transmission electron microscope (HRTEM) has been used to investigate the sample and to provide a formation of more ordered and crystalline structures.

\section{TEM Analysis}

\section{Crystallinity and graphite formation}

The following HRTEM images show a well-dispersed and aligned-CuNP of a $50 \mathrm{~nm}$ average diameter inside the fibers

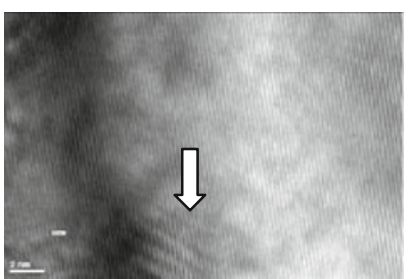

$\mathrm{H}=\mathbf{1} \mathbf{c m}$

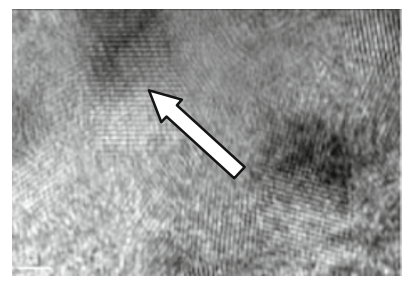

$\mathrm{H}=7 \mathrm{~cm}$
Fig. 5 Formation of $0.333 \mathrm{~nm}$ graphite lamellas for carbonized PAN samples

ranged from 67 to $293 \mathrm{~nm}$ diameters and on its surface as well. Also its diffraction pattern shows a full crystallinity for the heat treated nanofibril composite structure.

Figure 5 proved a formation of 2D graphitic structures (filled white arrows) of a graphitic lamellas of $0.333 \mathrm{~nm}$ thickness. The formation of such 2D graphitic structure can not be seen for the as electrospun samples.

For the carbonized samples a noticeable phenomenon has been observed as shown in Fig. 6a, b a formation of 3D graphitic structure (diamond like structure) has been captured as shown in the specified circles in many parts of the $\mathrm{CuNP} /$ carbon fibril composites specially at the edges of the fiber, speculation for such phenomenon may be explained based on two hypothesis: (a) due to the presence of the highly crystalline CuNP that forces the graphitic atoms to mimic its structure to minimize the state of energy (entropy part) of the atoms arrangement, or/and (b) due to the interaction between crystalline CuNP and PAN nanofiber during carbonization. It has been noticed that; the type of interaction increases as PAN nanofiber diameter decreases, so it is more likely has been found or/and seen with smaller fibril diameters.

\section{Conductivity measurements}

The mixing rule is a logical form for estimation of conductivity of composites. The most appropriate form of mixing rule can be written as:

$\sigma^{\alpha}=\left(1-V_{\mathrm{f}}\right) \sigma_{M}^{\alpha}+V_{\mathrm{f}} \sigma_{A}^{\alpha}$

where $\sigma_{M}, \sigma_{A}$ conductivity of the individual phase $M$ and $A$, $\alpha$ constant depends on the morphology of composite, $V_{\mathrm{f}}$ volume Fraction

Conductivity of CuNP/carbon single Nanofibril Composite

$\sigma_{\mathrm{SNFC}}=\left(1-V_{\mathrm{f}}\right) \sigma_{\mathrm{CNF}}+V_{\mathrm{f}} \sigma_{\mathrm{CuNP}}$

where $\sigma_{\mathrm{SNFC}}$ conductivity of single nanofibril composite, $\sigma_{\mathrm{CNF}}$ conductivity of carbon nanofiber, $\sigma_{\mathrm{CuNP}}$ conductivity of copper nanoparticle, $V_{\mathrm{f}} \mathrm{CuNP}$ volume fraction, it has been taken as function in $W_{\mathrm{f}} \%$ by using the following relationship $\mathrm{V}_{\mathrm{f}} \%$ has been correlated to $W_{\mathrm{f}} \%$ by using the 


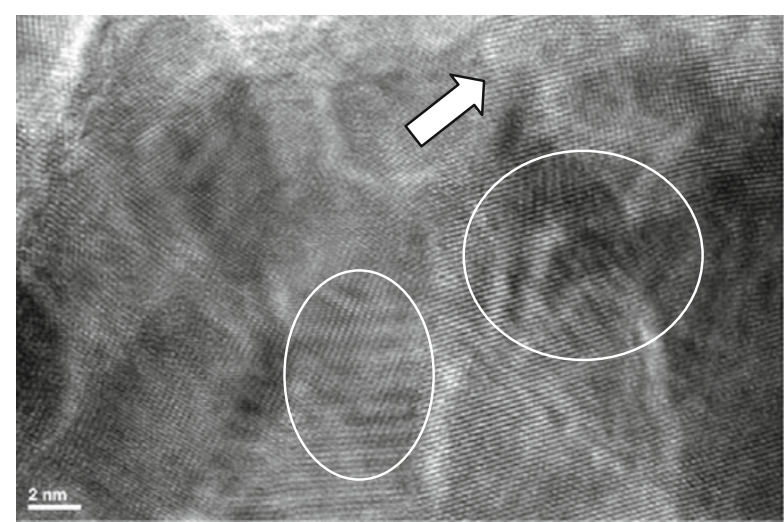

(a) $1 \mathrm{wt} \% \mathrm{CuNP}$ at $\mathrm{H}=1 \mathrm{~cm}$

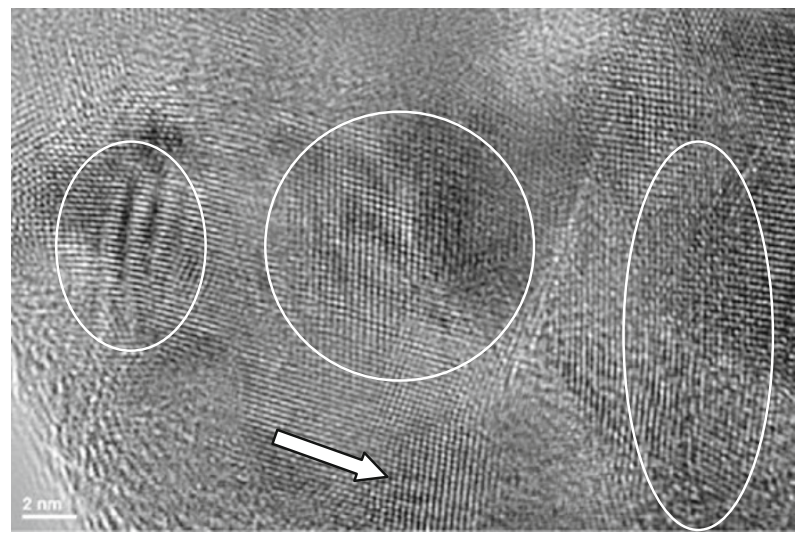

(b) $1 \mathrm{wt} \% \mathrm{CuNP}$ at $\mathrm{H}=7 \mathrm{~cm}$

Fig. 6 Formation of 3D nanostructures of CuNP/Carbon nanofibril composites

density of CuNP $=8.94 \mathrm{~g} / \mathrm{cm}^{3}$ and density of carbon nanofiber $=1.7 \mathrm{~g} / \mathrm{cm}^{3}$.

$\alpha=-1$ [values of $\alpha$ depends on the configuration between the two materials, for parallel arrangement $=1$, for series arrangement $=-1$ and in random arrangement between $-1 / 3$ and $2 / 3$, as CuNP is found to be in parallel with the nanofiber as it has been captured and shown in Fig. $7 \propto$ assumed to be equal -1 (Zakri et al. 1998)] (Fig. 8).

\section{Conductivity of CuNP/carbon nanofibril composite nonwoven fabrics}

$\sigma_{\mathrm{F}}=\left(1-V_{\mathrm{f}}\right) \sigma_{\mathrm{o}}+V_{\mathrm{f}} \sigma_{\mathrm{SNF}}$

where $\sigma_{\mathrm{F}}$ conductivity of the fabric, $\sigma_{\mathbf{o}}$ conductivity of vacuum, $\sigma_{\mathrm{SNF}}$ conductivity of single nanofibril composite $\alpha=\alpha_{1}\left(1-V_{\mathrm{f}}\right)+V_{\mathrm{f}} \alpha_{2}$

where $\alpha_{1}$ and $\alpha_{2}$ constant depends on the morphology of composite, in random mixture the values of conductivity are limited by upper and lower bound, based on that $\alpha_{1}$ and $\alpha_{2}$ values will be as follows: $\alpha_{1}=-1 / 3, \alpha_{2}=2 / 3$. Then

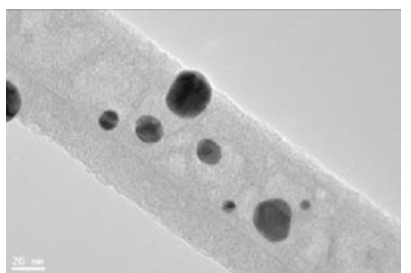

CuNP distribution

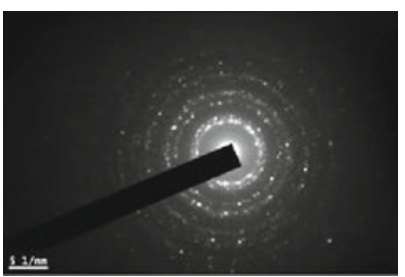

CuNP Diffraction Pattern
Fig. 7 CuNP distribution and diffraction patterns

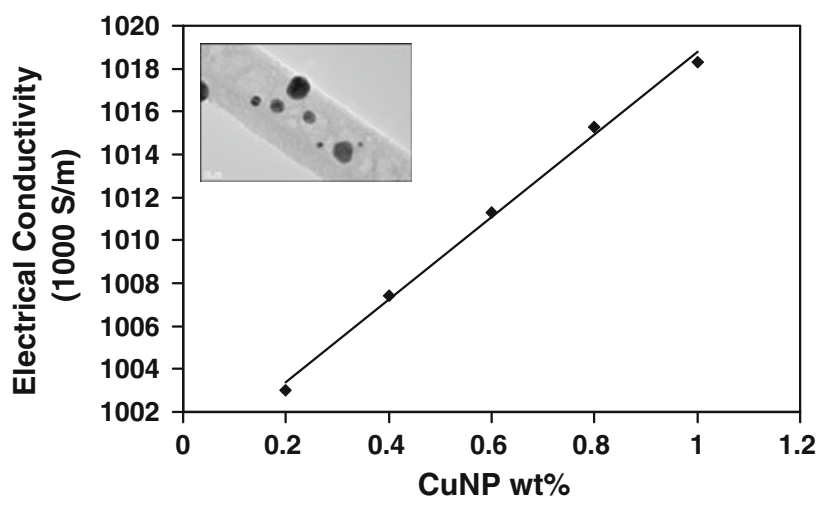

Fig. 8 Electrical conductivity of $\mathrm{CuNP} /$ carbon single nanofibril composites

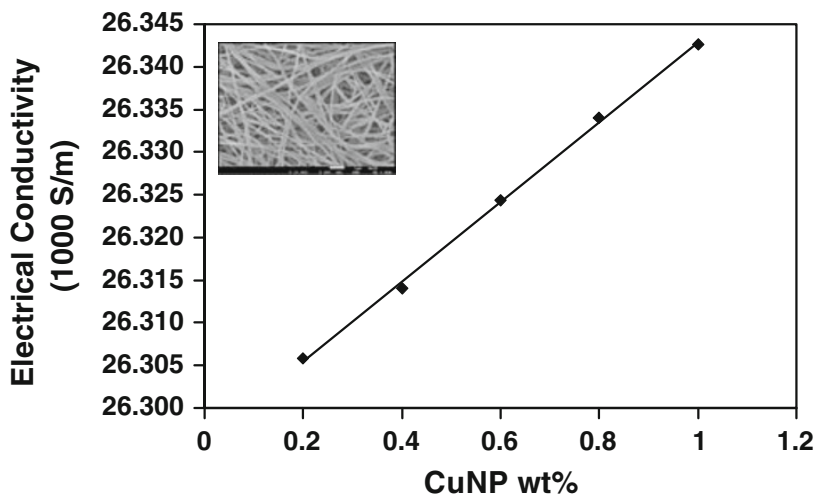

Fig. 9 Electrical conductivity of $\mathrm{CuNP} /$ carbon nanofibril composite nonwoven fabrics

$\alpha=0.186$ for $V_{\mathrm{f}}=0.508$ (fiber to whole fabric volume fraction) counted from randomly selected SEM images by using simple graphical soft ware (Fig. 9).

\section{Conclusions}

1. $64 \mathrm{~nm}$ minimum average PAN fiber diameter found to be collected at $7 \mathrm{~cm}$ spinning height and $23 \mathrm{kV}$ with 4 $\mu \mathrm{A}$ by using wet electrospinning set-up. 
2. Wet-electrospun PAN fibers and CuNP/PAN nanofibril composite diameters increases as spinning height decreases and wt\% of CuNP increases.

3. Electrical conductivity of CuNP/Carbon increases as CuNP wt $\%$ increases for both nanofibril composites and nonwoven nanofibroses fabrics.

4. Transformation from nonconductive CuNP/PAN nonwoven fabrics of $2 \times 10^{-4} \mathrm{~S} / \mathrm{m}$ to semi conductive $\mathrm{CuNP} /$ Carbon nonwoven fabrics of $2 \times 10^{4} \mathrm{~S} / \mathrm{m}$ has been reported.

5. A conductivity of $102 \times 10^{4} \mathrm{~S} / \mathrm{m}$ for a single CuNP/ Carbon nanofibril composite has been reported at $1 w t \%$ CuNP while for its nonwoven nanofibroses fabrics $2.6 \times 10^{4} \mathrm{~S} / \mathrm{m}$ has been reported.

6. Heat treatment of electrospun PAN fibers and CuNP/ PAN nanofibril composites showed a crystalline graphite structure and improvement in electrical conductivity of the whole fabric.

7. HRTEM images and diffraction patterns showed a formation of $3 \mathrm{D}$ ordered structure.

More investigations for the interaction between CuNP and graphitic lamella structures is essentially required to identify the exact formed structure type (on going research work).

Acknowledgments Authors would like to thank KAIN, Saudi Arabia for advanced characterizations of the samples. This work has been supported by CEREM, KSU Grant number 430-CEREM-01 and by KACST, Grant number 08-ADV171-2.

Open Access This article is distributed under the terms of the Creative Commons Attribution License which permits any use, distribution and reproduction in any medium, provided the original author(s) and source are credited.

\section{References}

Ali AA (2008) New generation of super absorber nanofibroses hybrid fabric by electro-spinning, synthesis, characterization and industrial applications of nanoparticles and nanostructure materials work shop hosted by MuCSAT and NSF, New Borg El-Arab, Alexandria, Egypt 11-15 November, 2005. J Mater Process Technol 199:193-198

Ali AA, Al-Asmari Kh A (2010) Morphological, Mechanical and Electrical Properties of Thermally Stable CNT/Carbon NanoFibril
Composite Structures, ICCE-18 Conference, Anchorage, Alaska, USA, pp 4-10

Ali AA, Geshury AJ, Ko FK (2002) Ultra-fine carbon fibers and fibrous structures from electro-spun PAN polymer solution. In: Fiber society annual tech. Conf, October 16-18, Natick, Massachusetts, USA

Ali AA, Rutledge GC (2009) Hot-Pressed Electrospun PAN nanoFibers: an Idea for Flexible Carbon Mat. J Mater Process Technol 209:4617-4620

Bal S (2010) Experimental study of mechanical and electrical properties of carbon nanofiber/epoxy composites. Mater Design 31:2406-2413

Doshi J, Reneker DH (1995) Electro-spinning process and application of electrospun fibers. J Electrostatic 35:51-160

Fan J, Wan M, Zhu D, Chang B, Pan Z, Xie S (1999) Synthesis, characterizations and physical properties of carbon nanotubes coated by conducting polypyrrole. J Appl Polymer Sci 74:26052610

Fang X, Reneker DH (1996) DNA fibers by electrospinning. J Polymer $1-8$

Guillou CL, Brunet F, Irifune T, Ohfuji H, Rouzaud JN (2007) Nanodiamond nucleation below 2,273 K at $15 \mathrm{GPa}$ from carbons with different structural organizations. Carbon 45:636-648

Kim HM, Kim K, Lee SJ, Joo J, Yoon HS, Cho SJ et al (2004) Charge transport properties of composites of multiwalled carbon nanotube with metal catalyst and polymer: application to electromagnetic interference shielding. Appl Phys 4:577-580

Ko F, Gogotsi Y, Ali AA, Naguib N, Ye H, Yang G, Li C, Willis P (2003) Electrospinning of continuous carbon nanotube-filled nanofiber yarns. Adv Mater 15:1161-1165

Koerner H, Liu W, Alexander M, Mirau P, Dowty H, Vaia RH (2005) Deformation-morphology correlations in electrically conductive CNT-thermoplastic polyurethane nanocomposites. Polymer 46: $4405-4420$

Mack J, Viculis L, Ali AA, Luoh R, Yang G, Hahn T, Ko F, Kaner R (2005) Graphite nanoplatelet reinforcement of electrospun polyacrylonitrile nanofibers. Advanced Materials 17:77-80

Pötschke P, Bhattacharyya AR, Janke A (2004) Carbon nanotubefilled polycarbonate composites produced by melt mixing and their use in blends with polyethylene. Carbon 42:965-969

Reneker DH, Chun I (1996) Nanometer diameter fibers of polymer, produced by electrospinning. Nanotechnology 7:216-223

Spitalsky Z, Tasis D, Konstantinos Papagelis K, Galiotis C (2010) Carbon nanotube-polymer composites. Chem Process Mech Electr Prop 35:357-401

Sun LT et al (2005) Synthesis and characterization of diamond nanowires from carbon nanotubes. Diam Relat Mater 14:749-752

Uvarov NF (2000) Estimation of composites conductivity using a general mixing rule Institute of Solid State Chemistry, Siberian Branch of Russian Academy of Science, Kutateladze. Solid State Ion 136-137:1267-1272

Zakri T, Laurent J-P, Vauclin M (1998) Theoretical evidence for 'Lichtenecker's mixture formulae' based on the effective medium theory. J Phys D Appl Phys 31:1589-1594 